\title{
LINEAR RESPONSE THEORY IN STOCHASTIC RESONANCE
}

\author{
M.I. Dykman ${ }^{1}$ \\ School of Physics and Materials, Lancaster University, Lancaster LA1 4YB, UK \\ H. Haken \\ Institut für Theoretische Physik und Synergetik, Universität Stuttgart, \\ D-70550 Stuttgart, Germany \\ Gang $\mathrm{Hu}$ \\ Department of Physics, Beijing Normal University, Beijing 100875, China \\ D.G. Luchinsky², R. Mannella ${ }^{3}$, P.V.E. McClintock \\ School of Physics and Materials, Lancaster University, Lancaster LA1 4YB, UK \\ C.Z. Ning \\ Institut für Theoretische Physik und Synergetik, Universität Stuttgart, \\ W-7000 Stuttgart 80, Germany \\ N.D. Stein \\ School of Physics and Materials, Lancaster University, Lancaster LA1 4YB, UK \\ N.G. Stocks \\ Department of Engineering, University of Warwick, Coventry CV4 7AL, UK.
}

\begin{abstract}
$\underline{\text { Abstract }}$
The susceptibility of an overdamped Markov system fluctuating in a bistable potential of general form is obtained by analytic solution of the Fokker-Planck equation (FPE) for low noise intensities. The results are discussed in the context of the LRT theory of stochastic resonance. They go over into recent results (Gang Hu et al Phys. Lett. A 172, 21, 1992) obtained from the FPE for the case of a symmetrical potential, and they coincide with the LRT results (Dykman et al, Phys. Rev. Lett. 65, 2606, 1990; JETP Lett 52, 144, 1990; Phys. Rev. Lett. 68, 2985, 1992) obtained for the general case of bistable systems.
\end{abstract}

PACS: $05.40+\mathrm{j}, 78.50$

\footnotetext{
${ }^{1}$ Permanent address: Department of Physics, Stanford University, Stanford, California 94305, USA

${ }^{2}$ Permanent address: Institute for Metrology, 117965 Moscow, Russia

${ }^{3}$ Permanent Address: Dipartimento di Fisica, Università di Pisa, 56100 Pisa, Italy
} 
The phenomenon of stochastic resonance (SR), where a periodic signal in a system and the signal-to-noise ratio are enhanced by external noise, has attracted much attention recently and by now has been observed in several physical systems (see [1] and references therein). The occurrence of SR is related to the nonlinearity of a system. At the same time, for small enough amplitude $A$ of the periodic force causing a signal, the amplitude of the signal is proportional to $A$, and therefore many aspects of SR can conveniently and effectively be analysed [2-6] in terms of standard linear response theory (LRT) [7]. The LRT analysis amounts to finding the susceptibility of the system $\chi(\Omega)$ and its dependence on the noise intensity $D$ and on the frequency $\Omega$ of the force. The susceptibility is defined in a standard way [7] as the coefficient of proportionality between the periodic term in the ensemble-averaged value of the coordinate $\delta\langle q(t)\rangle$ (the signal) and the force $A \exp (-i \Omega t)$,

$$
\delta\langle q(t)\rangle=\chi(\Omega) A \exp (-i \Omega t)
$$

(in the case of a nonstationary system the definition (1) has to be modified). The amplitude of the signal is equal to $|\chi(\Omega)| A$, and SR arises when $|\chi(\Omega)|$ increases with $D$ in some interval of $D$ (and then decreases again for higher $D$, so that the dependence on noise intensity is of the form of a resonant curve, thus justifying the term "stochastic resonance").

The LRT formulation is not confined to particular systems or particular types of noise and, indeed, was used to predict and observe SR [4,6] in systems drastically different from that performing Brownian motion in a symmetric double-well potential which is usually considered as the prototype of SR-displaying systems [1]. One of the advantageous features of this formulation is that, in the case of systems at thermal equilibrium or quasiequilibrium (in which case it is temperature $T$ that corresponds to the noise intensity $D$ ), the susceptibility $\chi(\Omega)$ is related, via the fluctuation-dissipation relations, to the spectral density of fluctuations (SDF) of the coordinate $q(t)$ [7]. Therefore, if the SDF is known from the experimental data, it is straightforward to predict whether the system will or will not display SR when driven by a force of a given frequency even without establishing the underlying physical mechanism; we note that the corresponding analysis does not assume that the system is Markovian or whatever - the only thing assumed is that it is in thermal equilibrium.

The theoretical evaluation of the susceptibility for some simple model noise-driven systems that display SR, and for systems performing Brownian motion in a double-well potential in particular, has been done in Refs. [2 - 6]. The analysis of bistable systems could be done for small noise intensities where there are two strongly different time scales that characterize the motion of a system: the relaxation time $t_{r}$ over which the system approaches a stable state (the longest of these times), and the reciprocal probabilities $W_{n m}^{-1}$ of fluctuational transitions $n \rightarrow m(n, m=1,2)$ between the states,

$$
W t_{r} \ll 1, \quad W=W_{12}+W_{21}
$$

Two approaches have been used. The first of these $[2,3]$ is based on the idea that, for low noise intensities, a system spends most of its time localized close to the stable states, and only occasionally do there occur fluctuational transitions between the states; therefore the susceptibility is a sum of the partial susceptibilities $\chi_{n}(\Omega)$ from small-amplitude vibrations about the stable positions $q_{n}(n=1,2)$, weighted with the populations $w_{n}$ of the stable states, and of the susceptibility related to the fluctuational transitions, $\chi_{t r}(\Omega)$, which 
describes the effect of the modulation of the transition probabilities and hence of the populations of the stable states by a periodic force (cf. [8]):

$$
\chi(\Omega)=\sum_{n=1,2} w_{n} \chi_{n}(\Omega)+\chi_{t r}(\Omega), \quad w_{n}=W_{m n} / W
$$

The evaluation of $\chi_{n}$ reduces to the solution of a linearized problem, and the explicit expression for $\chi_{t r}$ has been obtained for systems driven by Gaussian noise in the range $\Omega t_{r} \ll 1\left(\chi_{t r} \propto W / \Omega\right.$ for $W \ll \Omega$, cf. Eq.(18) below, and it becomes very small for $\Omega t_{r} \sim 1$ in the range (2)).

A different approach has been suggested in Ref.5 for the analysis of overdamped Brownian motion in a symmetrical bistable potential $U(q)$,

$$
\dot{q}+U^{\prime}(q)=f(t), \quad\left\langle f(t) f\left(t^{\prime}\right)\right\rangle=2 D \delta\left(t-t^{\prime}\right)
$$

The basic idea of this approach is to reduce the Fokker-Planck equation

$$
\frac{\partial P(q, t)}{\partial t}=\frac{\partial\left(U^{\prime}(q) P(q, t)\right)}{\partial q}+D \frac{\partial^{2} P(q, t)}{\partial q^{2}}
$$

that corresponds to the Langevin equation (4) to an eigenvalue problem and to express the susceptibility in terms of the matrix elements of the coordinate $q$ on the corresponding eigenfunctions. It was then possible to evaluate the susceptibility explicitly in the range of noise intensities $D$ where (2) holds. A systematic perturbation theory in the amplitude of a sinusoidal force has been developed for systems described by the Fokker-Planck equation and applied to SR in an effectively two-state approximation in Ref [9].

The purpose of the present paper is to demonstrate that the two approaches $[2,3]$ and [5] give identical results and to generalize the approach [5] to the case of an arbitrary double-well potential $U(q)$.

The reduction of Eq.(5) to a Hermitian eigenvalue problem is standard [10, 11]: one seeks the solution in the form

$$
\begin{gathered}
P(q, t)=Z^{-1 / 2} \exp (-U(q) / 2 D) \sum_{j=0}^{\infty}|j\rangle \exp (-\lambda(j) t), \quad|j\rangle \equiv \psi(j ; q), \\
Z=\int_{-\infty}^{\infty} \exp (-U(q) / D) d q
\end{gathered}
$$

and then arrives at a Schrodinger-type equation for the eigenfunctions $\psi(j ; q)$ :

$$
-D^{2} \psi^{\prime \prime}(j ; q)+V(q) \psi(j ; q)=D \lambda(j) \psi(j ; q), \quad V(q)=\frac{1}{4}\left(U^{\prime}(q)\right)^{2}-\frac{1}{2} D U^{\prime \prime}(q)
$$

The functions $\psi(j ; q)$ are orthonormal, $\left\langle j \mid j^{\prime}\right\rangle=\delta_{j j^{\prime}}$ (the form of the expansion (6) is a bit different from that used in [5]). Following the arguments given in Ref. 5 one can arrive at the following expression for the susceptibility of the system (4) with respect to a force $A \exp (-i \Omega t)$ :

$$
\chi(\Omega)=-2 \sum_{j}(\lambda(j)-i \Omega)^{-1}\langle 0|q| j\rangle\left\langle j\left|\frac{\partial}{\partial q}\right| 0\right\rangle \quad\left(\left\langle j|\hat{L}| j^{\prime}\right\rangle \equiv \int_{-\infty}^{\infty} d q \psi(j ; q) \hat{L} \psi\left(j^{\prime} ; q\right)\right)
$$


The eigenvalues $\lambda(j)$ and the eigenfunctions $\psi(j ; q)$ for low noise intensities $D$ were analysed in Ref. [11]. The qualitative results can be easily understood by noticing that, for a bistable initial potential $U(q)$ and small $D$, the potential $V(q)$ that appears in Eq. (7) has 3 minima of nearly the same depth placed close to the stable positions $q_{1,2}$ and an unstable stationary point $q_{s}$ (local maximum of $U(q) ; U^{\prime}\left(q_{1,2}\right)=U^{\prime}\left(q_{s}\right)=0, U^{\prime \prime}\left(q_{1,2}\right)>$ $\left.0, U^{\prime \prime}\left(q_{s}\right)<0\right)$. Close to the minima $V(q)$ is parabolic:

$$
\begin{aligned}
& V(q) \approx-\frac{1}{2} D U_{n}^{\prime \prime}+\frac{1}{4}\left(U_{n}^{\prime \prime}\right)^{2}\left(q-q_{n}\right)^{2}, \quad\left|q-q_{n}\right| \ll\left|q_{n}-q_{s}\right|, \quad U_{n}^{\prime \prime} \equiv U^{\prime \prime}\left(q_{n}\right) \\
& V(q) \approx \frac{1}{2} D\left|U_{s}^{\prime \prime}\right|+\frac{1}{4}\left(U_{s}^{\prime \prime}\right)^{2}\left(q-q_{s}\right)^{2}, \quad\left|q-q_{s}\right| \ll\left|q_{1,2}-q_{s}\right|, \quad U_{s}^{\prime \prime} \equiv U^{\prime \prime}\left(q_{s}\right)
\end{aligned}
$$

The lowest "energy levels" $\overline{\lambda_{n}}(0)$ of the motion within the wells $n=1,2$ of the potential $V$ (the overbar is used to indicate intrawell states, and the subscript enumerates the wells at the corresponding stable positions $q_{n}$ ) are degenerate, with $\bar{\lambda}_{1,2}(0)=0$ to the lowest order in $D$, giving rise to tunnelling splitting of these levels [11]. The low-lying excited intrawell states $\overline{|j\rangle_{n}} \equiv \overline{\psi_{n}}(j ; q)$ are those of quantum harmonic oscillators, and

$$
\overline{\lambda_{n}}(k) \approx k U_{n}^{\prime \prime}
$$

(and of course, high excited states cannot be prescribed to a particular well).

The exact wave function of the ground state (that allows for the tunnelling splitting) is known to be

$$
\psi(0 ; q)=Z^{-1 / 2} \exp (-U(q) / 2 D), \quad \lambda(0)=0
$$

It has sharp maxima at $q_{1,2}$ (of width $\delta q \sim\left(D / U^{\prime \prime}\left(q_{n}\right)\right)^{1 / 2}$ ) and a minimum at $q_{s}$. Near the maxima, the function (11) is close to the intrawell ground-state oscillatory functions $\overline{\psi_{n}}(0 ; q)$ weighted with the factors $Z^{-1 / 2}\left(2 \pi D / U_{n}^{\prime \prime}\right)^{1 / 4} \times \exp \left(-U\left(q_{n}\right) / 2 D\right)$. This makes it possible to evaluate the contribution to the susceptibility (8) from the excited intrawell states, $\chi_{\text {intra }}(\Omega)$. Since, for a harmonic oscillator, the only nonzero matrix elements of the operator $\partial / \partial q$ are those between adjacent states, only the first excited intrawell states, $\psi(j ; q) \approx \overline{\psi_{n}}(1 ; q)(n=1,2)$ will contribute to $\chi_{\text {intra }}(\Omega)$. Allowing for the explicit form of the matrix elements, known from quantum mechanics [12], and for Eqs.(9), (10) we get, with an accuracy to small corrections $\sim D$ :

$$
\chi_{\text {intra }}(\Omega) \approx \sum_{n=1,2} w_{n}\left(U_{n}^{\prime \prime}-i \Omega\right)^{-1}
$$

Here, $w_{n}$ is the population of the $n$th stable state,

$$
w_{n}=\frac{\exp \left(-\Delta U_{3-n} / D\right)\left(U_{3-n}^{\prime \prime}\right)^{1 / 2}}{\sum_{m=1,2} \exp \left(-\Delta U_{m} / D\right)\left(U_{m}^{\prime \prime}\right)^{1 / 2}}, \quad \Delta U_{m} \equiv U\left(q_{s}\right)-U\left(q_{m}\right)
$$

and we have evaluated the integral (6) for $Z$ by the steepest descent method. The overlap integral of the function $\psi(0 ; q)$ with (non-oscillatory) wave functions of highly excited states, as well as with the wave functions that correspond to the low-lying states in the well of the potential $V(q)$ centered at $q_{s}$, are exponentially small, and therefore the 
contribution from the corresponding matrix elements to the susceptibility (8) can be ignored.

The only other important contribution to $\chi(\Omega)$ besides $(12)$ is that from the matrix elements calculated between the function (11) and the function $\psi(1 ; q)$ corresponding to the excited state that results from the tunnel splitting of the degenerate intrawell state, whence

$$
\chi(\Omega) \approx \chi_{\text {intra }}(\Omega)+\chi_{\text {tun }}(\Omega), \quad \chi_{\text {tun }}(\Omega)=\frac{\lambda(1)}{D} \frac{|\langle 0|q| 1\rangle|^{2}}{\lambda(1)-i \Omega}
$$

Here, we have implemented the interrelation [11], well-known from quantum mechanics, between the matrix elements of the operators $\partial / \partial q$ and $q$ in terms of the energy difference between the states. The problem of evaluating $\chi_{\text {tun }}$ comes to the evaluation of the matrix elements of the coordinate. The latter can be done by making use of the explicit form of the wave functions $|0\rangle(11)$ and $|1\rangle$. The function $|1\rangle$ as well as the value of $\lambda(1)$ were found in [11]. An alternative way to find them is based on applying to the present problem the approach suggested in [12] to calculate tunnelling level splitting in a symmetrical doublewell potential. It is easy to see that, in the spirit of [12], the wave function $\psi(1 ; q)$ can be sought in the form

$$
\begin{gathered}
\psi(1 ; q)=A_{0} \psi(0 ; q)+A_{1} \tilde{\psi}(1 ; q) \\
\tilde{\psi}(1 ; q) \approx\left\{\begin{array}{cl}
-\psi(0 ; q), & q_{s}-q \gg\left(D /\left|U_{s}^{\prime \prime}\right|\right)^{1 / 2} \\
\psi(0 ; q)\left(2\left|U_{s}^{\prime \prime}\right| / \pi D\right)^{1 / 2} \int_{q_{s}}^{q} d q^{\prime} \exp \left[\frac{U\left(q^{\prime}\right)-U\left(q_{s}\right)}{D}\right], & \left|q-q_{s}\right| \lesssim\left(D /\left|U_{s}^{\prime \prime}\right|\right)^{1 / 2} \\
\psi(0 ; q), & q-q_{s} \gg\left(D /\left|U_{s}^{\prime \prime}\right|\right)^{1 / 2}
\end{array}\right.
\end{gathered}
$$

The coefficients $A_{0,1}$ can be obtained from the conditions of normalization of $\psi(1 ; q)$ and of orthogonality of $\psi(1 ; q)$ and $\psi(0 ; q)$, whereas the eigenvalue $\lambda_{1}$ is given by the expression

$$
\lambda(1)=-D \psi\left(0 ; q_{s}\right)\left(\frac{d \psi(1 ; q)}{d q}\right)_{q_{s}}\left[\int_{-\infty}^{q_{s}} d q \psi(0 ; q) \psi(1 ; q)\right]^{-1}
$$

that immediately follows from (7), (11).

It is seen from Eqs. (8), (14) - (16) that the susceptibility $\chi_{\text {tun }}(\Omega)$ due to the transitions to the tunnel-split state $0 \rightarrow 1$ is of the following form

$$
\begin{gathered}
\chi_{\text {tun }}(\Omega)=w_{1} w_{2}\left(q_{2}-q_{1}\right)^{2} \frac{\lambda(1)}{D}(\lambda(1)-i \Omega)^{-1} \\
\lambda(1)=\pi^{-1} \sum_{n=1,2}\left[\sqrt{U_{n}^{\prime \prime}\left|U_{s}^{\prime \prime}\right|} \exp \left(-\Delta U_{n} / D\right)\right]
\end{gathered}
$$

In the particular case of a symmetrical potential $U(q)$ Eqs.(1), (12), (14), (16) go over into the result obtained in [5].

It is interesting to compare the result of the present calculations with the expression (3) applied to the model (4). The explicit form of the susceptibility for this model was discussed in $[2,3]$. The intrawell susceptibilities $\chi_{n}(\Omega)=\left(U_{n}^{\prime \prime}-i \Omega\right)^{-1}$ follow from Eq.(4) linearized about the stable positions $q_{1,2}$. The expression for the susceptibility due to interwell transitions is of the form: 


$$
\chi_{t r}(\Omega)=w_{1} w_{2}\left(q_{2}-q_{1}\right)^{2} \frac{W}{D}(W-i \Omega)^{-1}
$$

where $w_{n}$ is the population of the $n$th state, and $W$ is the sum of the probabilities of the transitions between the states (cf. (2)). Allowing for the explicit expressions for the transition probabilities, one finds that the intrawell contribution $\chi_{\text {intra }}(\Omega)$ obtained above is precisely equal to that given by $(3)$, and that $\lambda(1)=W$ (cf. $[10,11]$ ) and therefore $\chi_{\text {tun }}(\Omega)=\chi_{\text {tr }}(\Omega)$, and the susceptibilities as a whole coincide with each other. So they do also in the particular case of a symmetric double-well potential discussed in [5]. The explicit expression for the spectral density of fluctuations considered in [5] coincides with that discussed in $[2,3]$ for the model (3) as well. We notice that, in the general case of an asymmetric potential, the intensity of the transition- (or, equivalently) tunnelling-induced peak in the susceptibility (17), (18) is proportional to $w_{1} w_{2} \propto \exp \left(-\left|U\left(q_{1}\right)-U\left(q_{2}\right)\right| / D\right)$, and it drops down extremely sharply with the difference in the depths of the wells.

It follows from the above discussion that two, seemingly different, approaches to the analysis of the susceptibility of a double-well system, one based on a simple physical picture of the motion and the other based on the solution of the Fokker-Planck equation when the latter applies, give identical results. This can be regarded as an extra indication of the usefulness of linear response theory in the context of stochastic resonance. 


\section{REFERENCES}

1. Special issue of the J. Stat. Phys. 70 (1993) no.1/2.

2. M. I. Dykman, R. Mannella, P. V. E. McClintock, and N. G. Stocks, Phys. Rev. Lett. 65 (1990) 2606; M. I. Dykman, P. V. E. McClintock, R. Mannella, and N. G. Stocks, Soviet Phys. JETP Lett. 52 (1990) 144.

3. M. I. Dykman, R. Mannella, P. V. E. McClintock, and N. G. Stocks, Phys. Rev. Lett. 68 (1992) 2985.

4. N.G. Stocks, N.D. Stein, S.M. Soskin, and P.V.E. McClintock, J. Phys. A 25 (1992) L1119.

5. G. Hu, H. Haken, and C.Z. Ning, Phys. Lett. A 172 (1992) 21.

6. M.I. Dykman, D.G. Luchinsky, R. Mannella, P.V.E. McClintock, N.D. Stein, and N.G. Stocks, J. Stat. Phys. 70 (1993) 463; ibid., 70 (1993) 479.

7. L.D. Landau and E.M.Lifshitz, Statistical Physics, 3rd edition (Pergamon, Oxford, 1980).

8. M.I.Dykman and M.A. Krivoglaz, Sov. Phys. JETP 50 (1979) 30; M.I. Dykman and M.A. Krivoglaz, in Soviet Physics Reviews, ed. by I.M. Khalatnikov (Harwood, New York 1984), Vol. 5, p.265.

9. Hu Gang, G. Nicolis and C. Nicolis, Phys. Rev. A 42, 2030 (1990).

10. N.G. van Kampen, Stochastic Processes in Physics and Chemistry (Elsevier, Amsterdam 1990); H. Risken, The Fokker-Planck Equation, 2nd edition (Springer-Verlag, Berlin 1989).

11. B. Caroli, C. Caroli, and B. Roulet, J. Stat. Phys. 21 (1979) 415.

12. L.D. Landau and E.M. Lifshitz, Quantum Mechanics, 3rd edition (Pergamon, Oxford 1977). 